\title{
COMPATIBILIDADE ENTRE ACARICIDAS E FERTILIZANTES FOLIARES EM FUNÇÃO DE DIFERENTES ÁGUAS NO CONTROLE DO ÁCARO DA LEPROSE DOS CITROS Brevipalpus phoenicis ${ }^{1}$
}

\author{
DANIEL JÚNIOR DE ANDRADE² ${ }^{2}$ MARCELO DA COSTA FERREIRA ${ }^{3}$, LUIZ GONZAGA FENÓLIO ${ }^{4}$
}

RESUMO - A adição de fertilizantes foliares à calda acaricida é frequentemente empregada na citricultura com o intuito de reduzir os custos das aplicações. Todavia, as implicações desta prática, na maioria dos casos, são desconhecidas. O objetivo do trabalho foi avaliar o efeito de caldas acaricidas em mistura com fertilizantes foliares e preparadas com diferentes águas no controle do ácaro B. phoenicis. Foram realizados dois experimentos em laboratório, nos anos de 2009 e 2010, utilizando-se de frutos de laranja para conter ácaros Brevipalpus phoenicis. Um dos experimentos constou de três bioensaios, nos quais se procurou verificar o efeito das misturas entre fertilizantes foliares e os acaricidas cyhexatin, propargite e acrinatrhrin sobre $B$. phoenicis. No outro experimento, além de verificar o efeito das misturas de fertilizantes com os acaricidas propargite e acrinatrhrin, buscou-se também avaliar o efeito de águas coletadas em diferentes fontes utilizadas no preparo das caldas sobre $B$. phoenicis. Os resultados evidenciaram que a aplicação dos fertilizantes foliares cloreto de zinco, cloreto de manganês, ureia e a mistura de fosfito de potássio + ureia + cloreto de zinco não afetaram a ação dos acaricidas cyhexatin, propargite e acrinathrin sobre o controle de $B$. phoenicis. As misturas dos cloretos de zinco e de manganês com o sulfato de magnésio e a adição de fosfito de potássio diminuíram a eficiência dos acaricidas propargite e acrinathrin, não devendo, a princípio, ser adicionadas numa mesma aplicação. Águas provenientes dos municípios paulistas de Itápolis, Pirangi e Pirassununga interferiram na ação dos acaricidas propargite e acrinathin sobre B. phoenicis, sendo que a água coletada em Itápolis apresentou resultados superiores em termos de eficiência. Verificaram-se alterações dos valores de $\mathrm{pH}$ e da condutividade elétrica após a adição de alguns dos fertilizantes à calda acaricida. Termos para indexação: CiLV, Citrus sinensis, Controle químico e pH.

\section{COMPATIBILITY BETWEEN ACARICIDES AND FOLIAR FERTILIZERS IN DIFFERENT WATER RESOURCES FOR CITRUS LEPROSIS MITE CONTROL Brevipalpus phoenicis}

\begin{abstract}
The addition of foliar fertilizers to an acaricide spray tank is frequently used in citrus crop targeting to reduce application costs. However, its implications are unknown. The aim of this study was evaluating the effect of acaricide spray mixed to foliar fertilizers and also prepared by different water resources for citrus mite control B. phoenicis. Two laboratory experiments were performed in 2009 and 2010, using B. phoenicis infested orange fruits. The first experiment was about three bio essays, which the purpose had been to evaluate the effect of leaf fertilizers and cyhexatin, propargite and acrinathrin acaricides blends into B. phoenicis control. In the second one, leaf fertilizers and propargite and acrinathrin acaricides blends were tested and, in addition, the evaluation of water resources effect into the spray preparation. The results presented that foliar fertilizers as zinc chloride; manganese chloride, urea and the mixture of potassium phosphite + urea + zinc chloride did not affect the cyhexatin, propargite and acrinathrin acaricide action into controlling B. phoenicis infestation. The zinc and manganese chloride mixtures with magnesium sulphate and the addition of potassium phosphite decreased some acaricides efficiency as propargite and acrinathrin, therefore, primarily, they must not be added at the same application. Water resources coming from Itápolis, Pirangi and Pirassununga interfered in the propargite and acrinathrin efficiency over B. phoenicis control, but the Itápolis water resource had shown better results. There was also observed a $\mathrm{pH}$ and electrical conductivity variation after some fertilizers addition into the spray water.
\end{abstract}

Index terms: CiLV, Citrus sinensis, Chemical Control and $\mathrm{pH}$.

\footnotetext{
1(Trabalho 172-12). Recebido em: 25-05-2012. Aceito para publicação em: 16-10-2012.

${ }^{2}$ Prof. Dr. Assistente Doutor do Departamento de Fitossanidade - FCAV/UNESP. Via de acesso Prof. Paulo Donato Castellane, $\mathrm{s} / \mathrm{n}$ CEP: 14884-970, Jaboticabal - SP. E-mail: danieldwv@yahoo.com.br

${ }^{3}$ Prof. Dr. Adjunto do Departamento de Fitossanidade - FCAV/UNESP. E-mail: mdacosta@fcav.unesp.br

${ }^{4}$ Mestre em Agronomia (Entomologia Agrícola) - FCAV/UNESP. E-mail: multipla.fenolio@terra.com.br
} 


\section{INTRODUÇÃO}

O Brasil é o principal país produtor de suco de laranja, sendo o Estado de São Paulo o maior produtor, com aproximadamente $80 \%$ da produção nacional de laranjas (AGRIANUAL, 2010). O sistema agroindustrial citrícola possui um papel socioeconômico fundamental para o agronegócio brasileiro, gerando em torno de 400 mil empregos diretos e 1,2 milhão de indiretos, correspondendo a aproximadamente $2 \%$ da mão de obra agrícola do País (AGRIANUAL, 2010; BOTEON; PAGLIUCA, 2010).

Contudo, a citricultura brasileira enfrenta diversas dificuldades, especialmente relacionadas ao aspecto fitossanitário. Entre as doenças, destaca-se a leprose dos citros, transmitida pelo ácaro Brevipalpus phoenicis (Geijkes, 1939), que é considerada uma das doenças virais de maior importância para a citricultura brasileira (BASTIANEL et al., 2010). O controle do ácaro-vetor com acaricidas tem sido praticamente a única tática de manejo da leprose nos pomares, sendo que os custos necessários para isso chegam a atingir US\$ 75 milhões por ano (KITAJIMA et al., 2010).

Pesquisas realizadas na década de 90 junto a diversos citricultores, técnicos pertencentes a cooperativas, mostraram mais de 120 combinações de misturas de tanque durante a aplicação de acaricidas na citricultura (ANDRADE, 1997), envolvendo principalmente acaricidas com inseticidas, fungicidas, fertilizantes foliares, óleos e adjuvantes.

$\mathrm{Na}$ agricultura, as misturas de tanque de produtos fitossanitários, juntamente com fertilizantes, são realizadas com o intuito de reduzir os custos das aplicações (HOUGHTON, 1982). Contudo, a adição de fertilizantes foliares à calda acaricida pode alterar substancialmente o efeito do acaricida sobre a praga-alvo, devido principalmente às alterações provocadas no $\mathrm{pH}$ da calda (BAUR et al., 1971; MOXNESS; LYM, 1989; MATUO; MATUO, 1995) e na condutividade elétrica (ELL-ATTAL et al., 1984; FARGHALAY et al., 2009), bem como pela possível incompatibilidade entre os produtos (HOUGHTON, 1982)

Internacionalmente, a preocupação dos pesquisadores e técnicos sobre os efeitos causados pelas misturas de tanque é relativamente antiga (SCHENCK; ADLERZ, 1962; PRADO et al., 2011). Em 1980, na Philadelfia - EUA, foi realizada a primeira conferência sobre aplicações de produtos fitossanitários com mistura de tanque, nos quais diversos pesquisadores se reuniram para discutir sobre os principais efeitos das misturas, especialmente aquelas envolvendo fertilizantes, devido às maiores alterações das características físico-químicas ocorridas na calda (WRIGHT et al., 1982). Além disso, a presença de impurezas e substâncias dissolvidas na água geralmente variam em função da fonte de coleta da água, podendo ou não interferir na ação de produto fitossanitário, quando utilizada como diluente para o preparo da calda de pulverização. Assim, a qualidade química da água, em relação ao $\mathrm{pH}$, sais e íons dissolvidos, é um fator a ser considerado no tratamento fitossanitário (HOUGHTON, 1982; PRADO et al., 2011).

Dessa forma, o objetivo do trabalho foi avaliar o efeito de caldas acaricidas em mistura com fertilizantes foliares e preparadas com diferentes águas no controle do ácaro $B$. phoenicis.

\section{MATERIAL E MÉTODOS}

Os experimentos foram realizados em 2009 e 2010, no Laboratório de Acarologia do Departamento de Fitossanidade da UNESP, Câmpus de Jaboticabal-SP.

Para a criação-estoque de B. phoenicis, foram utilizados frutos de laranja 'Pera' com verrugose, causada pelo fungo Elsinoe fawcetti (Bitancourt e Jenkis), colhidos em pomar do Câmpus da UNESP, sem aplicação de produtos fitossanitários, para a manutenção dos ácaros em laboratório. Esses frutos foram parcialmente parafinados, deixando-se uma arena sem parafina e circundada com cola entomológica para conter os ácaros, de acordo com a metodologia de Albuquerque et al. (1997). Os frutos com os ácaros foram mantidos em câmara climatizada à temperatura de $25^{\circ} \mathrm{C}$, umidade relativa de $70 \%$ e fotofase de 12 horas.

Nos experimentos, foram utilizados frutos de laranja da mesma origem dos utilizados para a criação do ácaro B. phoenicis. Após serem colhidos, os frutos foram lavados em água corrente e secos à sombra. Posteriormente, foram parcialmente parafinados, deixando-se uma área circular de $2,5 \mathrm{~cm}$ de diâmetro sem parafina, que foi circundada com cola entomológica, com o intuito de conter os ácaros. Em ambos os experimentos, os frutos foram mantidos em câmara climatizada nas mesmas condições utilizadas para a criação- estoque de $B$. phoenicis.

Mortalidade de $\boldsymbol{B}$. phoenicis em função da adição de fertilizantes foliares à calda acaricida - Foram realizados três bioensaios para avaliar as caldas acaricidas de cyhexatin (Sipcatin ${ }^{\circledR} 500$ SC), propargite (Omite $\left.{ }^{\circledR} 720 \mathrm{CE}\right)$ e acrinathrin (Rufast ${ }^{\circledR}$ 50 SC) com adição de fertilizantes foliares. Os 
fertilizantes foliares utilizados foram selecionados com base em entrevistas com citricultores da região Itápolis-SP, para conhecimento dos fertilizantes, das misturas e das dosagens utilizadas cotidianamente. Após as entrevistas, os tratamentos utilizados nos bioensaios foram definidos de acordo com as misturas mais praticadas pelos citricultores entrevistados. Os fertilizantes foliares selecionados foram: cloreto de zinco $\left(\mathrm{ZnCl}_{2}\right)$, cloreto de manganês $\left(\mathrm{MnCl}_{2}\right)$, fosfito de potássio $\left(\mathrm{KH}_{2} \mathrm{PO}_{3}\right)$, sulfato de magnésio $\left(\mathrm{MgSO}_{4}\right)$ e ureia $\left(\mathrm{NH}_{2}\right)_{2} \mathrm{CO}$. Para o preparo das caldas fitossanitárias, utilizou-se de água coletada em açude no Câmpus da UNESP.

O delineamento experimental adotado nos bioensaios foi o inteiramente casualizado, com oito tratamentos e uma testemunha com aplicação de água destilada, com oito repetições em cada tratamento, sendo cada repetição composta por um fruto de laranja (Tabela 1).

Imediatamente após o preparo das caldas acaricidas, determinaram-se o $\mathrm{pH}$, a condutividade elétrica, o oxigênio dissolvido e a temperatura das caldas. Em seguida, as caldas acaricidas foram aplicadas sobre os frutos de laranja em torre de Potter, calibrada a $4 \mathrm{lbf} / \mathrm{pol}^{2}$, aplicando-se $2 \mathrm{~mL}$ de calda por fruto, além do ponto de saturação da superfície tratada, com o intuito de proporcionar uma cobertura uniforme sobre os frutos. Após as aplicações, aguardou-se a secagem da calda acaricida sobre os frutos e, na sequência, transferiram-se para cada fruto dez ácaros fêmeas de $B$. phoenicis, procedentes da criação- estoque. Para realizar as transferências dos ácaros, empregaram-se um pincel de um pelo e microscópio estereoscópio, colocando-os na arena não parafinada e circundada pela cola entomológica. As avaliações de mortalidade de B. phoenicis foram realizadas a 1;3 e 5 dias após a transferência dos ácaros para os frutos.

Mortalidade de $B$. phoenicis em função da água de diferentes origens utilizadas no preparo das caldas acaricidas com fertilizantes foliares Este experimento foi realizado após a obtenção dos resultados do primeiro experimento, no qual foram utilizados os fertilizantes foliares que mais interferiram na eficiência dos acaricidas. $\mathrm{O}$ delineamento experimental adotado foi o inteiramente casualizado, em esquema fatorial $2 \times 3 \times 4+3$ testemunhas, totalizando 27 tratamentos, que foram repetidos quatro vezes, sendo cada repetição composta por um fruto de laranja. Os fatores empregados foram dois acaricidas (propargite e acrinathrin), água de três municípios do Estado de São Paulo (Pirassununga, Pirangi e Itápolis) e os fertilizantes foliares fosfito de potássio, sulfato de magnésio, a mistura entre cloreto de zinco e cloreto de manganês, e sulfato de magnésio, sem adição de fertilizantes. As testemunhas foram água destilada, água destilada com propargite e água destilada com acrinathrin.

As dosagens dos produtos utilizados, expressas em $\mathrm{mL}$ ou grama de produto comercial por $100 \mathrm{~L}$ de água, foram: propargite a $100 \mathrm{~mL}$, acrinathrin a 10 $\mathrm{mL}$, fosfito de potássio $\left(\mathrm{KH}_{2} \mathrm{PO}_{3}\right)$ a $150 \mathrm{~mL}$, sulfato de magnésio $\left(\mathrm{MgSO}_{4}\right)$ a $500 \mathrm{~g}$, cloreto de zinco $\left(\mathrm{ZnCl}_{2}\right)$ a $50 \mathrm{~mL}$ e cloreto de manganês $\left(\mathrm{MnCl}_{2}\right)$ a $50 \mathrm{~mL}$. As águas empregadas no preparo das caldas foram coletadas em poços artesianos localizados no sítio Cabeceira do Salto Grande - Pirassununga-SP, e na Fazenda Tabarana - Pirangi - SP, e em um açude localizado na Fazenda São Lourenço - Itápolis - SP.

Após o preparo das caldas, determinaram-se o $\mathrm{pH}$, a condutividade elétrica, o oxigênio dissolvido e a temperatura das caldas. Em seguida, as caldas acaricidas foram aplicadas sobre os frutos de laranja em torre de Potter, calibrada a $4 \mathrm{lbf} / \mathrm{pol}^{2}$, aplicando-se $2 \mathrm{~mL}$ de calda por fruto, além do ponto de saturação da superfície tratada, com o intuito de proporcionar uma cobertura uniforme sobre os frutos. Da mesma forma que no primeiro experimento, aguardou-se a secagem da calda acaricida e, em seguida, transferiram-se dez ácaros fêmeas de $B$. phoenicis para cada fruto, procedentes da criação-estoque. Para realizar as transferências dos ácaros, empregou-se um pincel de um pelo, sob microscópio estereoscópico, colocando-os na arena não parafinada e circundada pela cola entomológica. As avaliações de mortalidade de $B$. phoenicis foram realizadas a 1; 3 e 5 dias após a transferência dos ácaros para os frutos.

Os dados de número de ácaros, em ambos os experimentos, foram transformados em $\ln (x+5)$, para serem analisados pelo teste $\mathrm{F}$. As médias foram comparadas pelo teste de Tukey, a 5\% de probabilidade. A redução real, ou a eficiência,foi calculada pela fórmula de Abbott (1925), tomando-se por base a população de ácaros vivos de cada tratamento e transformados em porcentagem de sobrevivência. No segundo experimento, a porcentagem de sobrevivência antes de ser submetida à análise de variância foi transformada em:

$$
\operatorname{arco-seno} \sqrt{\frac{\mathrm{x}+1}{100}}
$$




\section{RESULTADOS E DISCUSSÃO}

Mortalidade de B. phoenicis em função da adição de fertilizantes foliares à calda acaricida - Para o acaricida cyhexatin, a um e três dias após a aplicação, verificou-se que o número de ácaros vivos de $B$. phoenicis e retidos na cola não apresentaram diferenças significativas entre os tratamentos com e sem adição de fertilizantes foliares (Tabela 2). Entretanto, para o propargite adicionado de cloreto de manganês, constatou-se que, um dia após a aplicação, houve menor número de ácaros vivos, diferindo significativamente da testemunha. Os demais tratamentos, na primeira avaliação após a aplicação, não deferiram significativamente da testemunha (Tabela 3). Aos três e cinco dias da aplicação, todos os tratamentos com propargite diferiram da testemunha em relação ao número de ácaros $B$. phoenicis vivos. Neste contexto, Campos Neto et al. (1993) verificaram que o propargite aplicado em mistura com enxofre apresentou eficiência inferior sobre o ácaro $B$. phoenicis quando comparada à eficiência do propargite aplicado isoladamente.

Em relação à fuga dos ácaros para a barreira adesiva, houve diferença significativa da calda de propargite com adição de fosfito de potássio em relação à testemunha, aos três dias após as aplicações, evidenciando maior repelência do ácaro pelo produto, todavia este fato não implicou diferenças significativas nas demais avaliações em relação aos demais tratamentos (Tabela 3 ).

Os tratamentos com o acaricida acrinathrin não apresentaram diferenças significativas entre si quanto ao número de ácaros vivos. Nas três avaliações realizadas após a aplicação, todos os tratamentos deferiram da testemunha que recebeu água destilada (Tabela 4). Contudo, houve diferença entre os tratamentos e a testemunha para os ácaros retidos na cola, em todas as avaliações, nos quais os tratamentos com acrinathrin apresentaram maior número de ácaros na cola, possivelmente devido ao efeito de irritabilidade e repelência deste acaricida causada sobre B. phoenicis (Tabela 4).

Todos os tratamentos com cyhexatin, aos três dias após a aplicação, apresentaram $100 \%$ de eficiência sobre $B$. phoenicis, independentemente da adição de fertilizante foliar. Observou-se que o tratamento com propargite e sulfato de magnésio apresentou 94,1\% de eficiência aos cinco dias da aplicação, enquanto nesta mesma avaliação os demais tratamentos com propargite apresentaram $100 \%$ de eficiência. Em contrapartida, os tratamentos com acrinathrin + fosfito de potássio, acrinathrin + sulfato de magnésio e acrinathrin + cloreto de zinco
+ cloreto de manganês + sulfato de magnésio, aos cinco dias após a aplicação, não atingiram $100 \%$ de eficiência, ficando aquém dos demais tratamentos com acrinathrin (Tabela 5).

Schenck e Adlerz (1962) avaliaram 72 combinações de inseticidas e fungicidas em mistura com fertilizantes foliares em cultura de melancia. Os autores verificaram que o controle de lagartas que se alimentam da casca da melancia, como Spodoptera exigua (Hübner, 1808) e Spodoptera frugiperda (Smith, 1797), foi melhor com o inseticida guthion em mistura com os fertilizantes foliares ureia ou nitrato de sódio e potássio. Jones e Kelsheimer (1968) estudaram a compatibilidade de diversos produtos fitossanitários na cultura do tomate. Estes autores constataram que o sulfato de zinco $\left(\mathrm{ZnSO}_{4}\right)$ em mistura com o fungicida M-45 proporcionou controle mais eficiente da mancha foliar cinzenta do tomate causada pelo fungo Stemphylium solani (Weber, 1930).

Pode-se verificar que ocorreram variações consideráveis dos valores de $\mathrm{pH}$ e da condutividade elétrica das caldas após a adição dos fertilizantes foliares; entretanto, nas condições do presente trabalho, tais alterações não interferiram na eficiência dos acaricidas sobre B. phoenicis (Tabela 6). Esses resultados concordaram com Andrade (1997), que constatou que os acaricidas hexithiazox, óxido de fenbutatina e dicofol não foram influenciados pelo $\mathrm{pH}$ da calda (3; 6 e 9) quanto às suas ações acaricidas sobre $B$. phoenicis.

Entretanto, o pH da água pode interferir na ação de um ingrediente ativo, pois altas concentrações de íons $\mathrm{H}+$ ou $\mathrm{OH}-$ poderão reagir com o ingrediente ativo, diminuindo, assim, a concentração deste na calda (PRADO et al., 2011). O acréscimo de produtos fitossanitários à água tende a alterar os valores de $\mathrm{pH}$ da calda de pulverização. Esses valores de $\mathrm{pH}$ influenciam na eficiência dos produtos utilizados, além de interferir nos níveis de dissociação dos ingredientes ativos e na estabilidade física das soluções (PRADO et al., 2011).

Por outro lado, com relação à condutividade elétrica de caldas fitossanitárias, El-Attal et al. (1984) afirmaram que o aumento da condutividade elétrica devido à adição de fertilizantes foliares proporcionou controle mais eficiente da lagarta Spodoptera littoralis (Boisduval, 1833), para a cultura do algodão.

A adição dos fertilizantes fosfito de potássio, ureia e cloreto de zinco, em mistura com os acaricidas, foi o tratamento que resultou nos menores valores de $\mathrm{pH}$. Entre os fertilizantes, constatou-se que o fosfito de potássio foi o que causou a maior diminuição do $\mathrm{pH}$ da calda, quando comparado à 
calda preparada somente com os acaricidas (Tabela 6). De acordo com Tawfik e El-Sisi (1987) e El-Sisi et al. (1989), as caldas fitossanitárias com o menores valores de $\mathrm{pH}$ e maiores de condutividade elétrica aumentam a atração entre as partículas da solução e a superfície da planta tratada.

Além disso, Simkiss e Mason (1983) mencionam que reduções drásticas do $\mathrm{pH}$ da calda fitossanitária podem provocar alterações na taxa respiratória de insetos e moluscos, causando aumento significativo da mortalidade. Esta constatação foi confirmada também por Farghaly et al. (2009) e Sayed et al. (2011). Neste sentido, Tawfik e El-Sisi (1987) verificaram que o aumento da condutividade elétrica da calda fitossanitária causou maior mortalidade da cochonilha Parlatoria ziziphus (Lucas, 1853), devido à maior deposição e penetração das partículas do ingrediente ativo presentes na calda.

El-Attal et al. (1984) realizaram trabalhos para a verificação do efeito de inseticidas em mistura com fertilizantes foliares contra lagartas de quarto instar de S. littoralis e constaram que houve efeito sinérgico entre as misturas de inseticidas e fertilizantes foliares. Os autores atribuíram este efeito sinérgico à presença de íons (deionização) na solução devido ao abaixamento do $\mathrm{pH}$ causado pelos fertilizantes, resultando em aumento da taxa de penetração do inseticida nas membranas internas da lagarta $S$. littoralis.

\section{Mortalidade de B. phoenicis em função da água de diferentes origens utilizadas no preparo das caldas acaricidas com fertilizantes foliares - Observou-se que, aos 3 e 5 dias após a transferência dos ácaros, houve interação entre os fatores acari- cidas, águas e fertilizantes foliares para número de ácaros vivos e porcentagem de sobrevivência (Tabela 7). Esses resultados indicaram que os fatores estuda- dos atuaram conjuntamente sobre a mortalidade do ácaro B. phoenicis.}

Verificou-se que o fator acaricida (A) apresentou significância nas avaliações realizadas aos três e cinco dias após a aplicação para número de ácaros vivos e porcentagem de sobrevivência (Tabela 7). Na avaliação realizada um dia após a aplicação, a calda preparada com água de Itápolis e com o fosfito de potássio apresentou a menor porcentagem de ácaros sobreviventes comparada às caldas com as águas de Pirangi e Pirassununga e com o mesmo fertilizante (Tabela 8).

Nas avaliações a um e cinco dias após a aplicação, as caldas com água de Pirangi, independentemente da adição ou não de fertilizantes, não apresentaram diferença significativa em relação à porcentagem de sobrevivência de ácaros. Em con- trapartida, aos cinco dias da aplicação, constatou-se que o tratamento com água de Itápolis, com adição de cloreto de zinco, cloreto de manganês e sulfato de magnésio, foi aquele no qual se obteve a menor porcentagem de sobrevivência de ácaros. Aos cinco dias da aplicação, observou-se, também, que o tratamento com água de Pirassununga sem adição de fertilizante foi o que apresentou a menor porcentagem de sobrevivência de $B$. phoenicis (Tabela 8).

De modo geral, os tratamentos com propargite apresentaram eficiência superior aos tratamentos com acrinathrin, independentemente da adição de fertilizantes e da água empregada no preparo das caldas (Tabela 9). Embora a adição dos fertilizantes cloreto de zinco, cloreto de manganês e sulfato de magnésio tenha promovido maior eficiência do acrinathrin, esta ainda não alcançou níveis satisfatórios de mortalidade do ácaro. Aos cinco dias da aplicação, com exceção da calda preparada com propargite e água de Pirangi e sem adição de fertilizante, assim como o propargite preparado em água destilada, que apresentaram percentuais de eficiência de $88,8 \%$ e $96,4 \%$, respectivamente, os demais tratamentos com propargite apresentaram $100 \%$ de eficiência (Tabela 9).

As variações de $\mathrm{pH}$ das águas utilizadas pode explicar, em parte, as diferenças relacionadas à sobrevivência dos ácaros. A água coletada em Pirassununga foi a mais alcalina com valor de $\mathrm{pH}$ de 8,2, enquanto as águas de Pirangi e Itápolis apresentaram $\mathrm{pH}$ de 7,7 e 6,4, respectivamente. Tundisi (1981) avaliou o $\mathrm{pH}$ de 52 reservatórios artificiais de água localizados no Estado de São Paulo, verificando que este variou de 5,5 a 7,9.

Reeves (1983) afirma que águas com valores de $\mathrm{pH}$ altos podem provocar hidrólise alcalina de determinados produtos fitossanitários, resultando em perdas de eficiência. De acordo com o autor, os inseticidas e acaricidas são mais suscetíveis à hidrólise do que os fungicidas, herbicidas e reguladores de crescimento. Afirma ainda que a perda de eficiência do produto é irreversível. Al-mughrabi e Nazer (1991) verificaram que o inseticida dimetoato, preparado com calda com $\mathrm{pH}$ de 8,4 , sofre perda de eficiência de 40,5\% após 24 horas, em pH 6,0; corrigido com ácido fosfórico, a perda passa a ser de $7,7 \%$ no mesmo período.

$\mathrm{O}$ efeito de misturas de inseticidas com adjuvantes sobre a lagarta $S$. littoralis e sobre algumas propriedades da calda foi estudado por Betana et al. (2004). Estes autores verificaram que todos os adjuvantes utilizados, entre os quais o ácido fosfórico e o ácido acético, alteraram as propriedades físico-químicas da água; além disso, algumas mis- 
TABELA 2 - Número médio de ácaros vivos Brevipalpus phoenicis para as diferentes caldas do acaricida cyhexatin com adição de fertilizantes foliares.

\begin{tabular}{|c|c|c|c|c|c|}
\hline \multirow{3}{*}{ Tratamentos } & \multirow{3}{*}{$\begin{array}{c}\text { Dosagens } \\
\text { (g ou } \mathrm{mL} \text { p.c./100 L) }\end{array}$} & \multicolumn{4}{|c|}{ Média de ácaros ${ }^{1}$} \\
\hline & & \multicolumn{2}{|c|}{ Vivos } & \multicolumn{2}{|c|}{ Retidos na cola } \\
\hline & & $1 \mathrm{DAT}^{2}$ & 3 DAT & 1 DAT & 3 DAT \\
\hline Cyhexatin (Cy) & 50 & $1,6 b^{3}$ & $1,6 \mathrm{~b}$ & $1,6 \mathrm{a}$ & $1,6 \mathrm{a}$ \\
\hline $\mathrm{Cy}+{ }^{4} \mathrm{ZnCl}_{2}$ & $50+50$ & $1,6 \mathrm{~b}$ & $1,6 \mathrm{~b}$ & $1,7 \mathrm{a}$ & $1,7 \mathrm{a}$ \\
\hline $\mathrm{Cy}+{ }^{5} \mathrm{MnCl}_{2}$ & $50+50$ & $1,7 \mathrm{~b}$ & $1,6 \mathrm{~b}$ & $1,7 \mathrm{a}$ & $1,7 \mathrm{a}$ \\
\hline $\mathrm{Cy}+{ }^{6} \mathrm{KH}_{2} \mathrm{PO}_{3}$ & $50+150$ & $1,7 \mathrm{~b}$ & $1,6 \mathrm{~b}$ & $1,7 \mathrm{a}$ & $1,7 \mathrm{a}$ \\
\hline $\mathrm{Cy}+{ }^{7} \mathrm{MgSO}_{4}$ & $50+500$ & $1,8 \mathrm{~b}$ & $1,6 \mathrm{~b}$ & $1,6 \mathrm{a}$ & $1,6 \mathrm{a}$ \\
\hline $\mathrm{Cy}+{ }^{8}\left(\mathrm{NH}_{2}\right)_{2} \mathrm{CO}$ & $50+300$ & $1,7 \mathrm{~b}$ & $1,6 \mathrm{~b}$ & $1,6 \mathrm{a}$ & $1,6 \mathrm{a}$ \\
\hline $\mathrm{Cy}+\mathrm{ZnCl}_{2}+\mathrm{MnCl}_{2}+\mathrm{MgSO}_{4}$ & $50+50+50+500$ & $1,6 \mathrm{~b}$ & $1,6 \mathrm{~b}$ & $1,6 \mathrm{a}$ & $1,6 \mathrm{a}$ \\
\hline $\mathrm{Cy}+\mathrm{KH}_{2} \mathrm{PO}_{3}+\left(\mathrm{NH}_{2}\right)_{2} \mathrm{CO}+\mathrm{ZnCl}_{2}$ & $50+150+300+50$ & $1,6 \mathrm{~b}$ & $1,6 \mathrm{~b}$ & $1,6 \mathrm{a}$ & $1,6 \mathrm{a}$ \\
\hline Testemunha (água destilada) & - & $2,6 \mathrm{a}$ & $2,5 \mathrm{a}$ & $1,6 \mathrm{a}$ & $1,7 \mathrm{a}$ \\
\hline Teste F & & $35,2 * *$ & $460,4 * *$ & $1,1^{\mathrm{NS}}$ & $1,0^{\mathrm{NS}}$ \\
\hline CV (\%) & & 8,6 & 2,2 & 5,6 & 5,7 \\
\hline
\end{tabular}

NS-não significativo; $(* *)$ Significativo a $1 \%$ de probabilidade; ${ }^{1}$ Dados originais transformados em $\ln (\mathrm{x}+5)$.

${ }^{2}$ Dias após o tratamento (DAT).$^{3}$ Letras iguais na mesma coluna não diferem entre si, pelo teste de Tukey $(\mathrm{p}<0,05) .{ }^{4} \mathrm{Cloreto}$ de zinco; ${ }^{5}$ Cloreto de manganês; ${ }^{6}$ Fosfito de potássio; ${ }^{7}$ Sulfato de magnésio e ${ }^{8}$ Ureia.

TABELA 3 - Número médio de ácaros vivos Brevipalpus phoenicis para as diferentes caldas do acaricida propargite com adição de fertilizantes foliares.

\begin{tabular}{|c|c|c|c|c|c|c|c|}
\hline \multirow{3}{*}{ Tratamentos } & \multirow{3}{*}{$\begin{array}{c}\text { Dosagens } \\
\text { g ou } \mathrm{mL} \text { p.c./100 L }\end{array}$} & \multicolumn{6}{|c|}{ Média de ácaros ${ }^{1}$} \\
\hline & & \multicolumn{3}{|c|}{ Vivos } & \multicolumn{3}{|c|}{ Retidos na cola } \\
\hline & & $1 \mathrm{DAT}^{2}$ & 3 DAT & 5 DAT & 1 DAT & 3 DAT & 5 DAT \\
\hline Propargite (Pr) & 100 & $2,6 \mathrm{a}$ & $1,7 \mathrm{~b}$ & $1,6 \mathrm{~b}$ & $1,6 \mathrm{a}$ & $1,8 \mathrm{ab}$ & $1,8 \mathrm{a}$ \\
\hline $\operatorname{Pr}+{ }^{4} \mathrm{ZnCl}_{2}$ & $100+50$ & $2,5 \mathrm{a}$ & $1,6 \mathrm{~b}$ & $1,6 \mathrm{~b}$ & $1,6 \mathrm{a}$ & $1,8 \mathrm{ab}$ & $1,8 \mathrm{a}$ \\
\hline $\operatorname{Pr}+{ }^{5} \mathrm{MnCl}_{2}$ & $100+50$ & $2,3 \mathrm{~b}$ & $1,6 \mathrm{~b}$ & $1,6 \mathrm{~b}$ & $1,8 \mathrm{a}$ & $1,8 \mathrm{ab}$ & $1,8 \mathrm{a}$ \\
\hline $\mathrm{Pr}+{ }^{6} \mathrm{KH}_{2} \mathrm{PO}_{3}$ & $100+150$ & $2,4 \mathrm{ab}$ & $1,7 \mathrm{~b}$ & $1,6 \mathrm{~b}$ & $1,6 \mathrm{a}$ & $2,0 \mathrm{a}$ & $2,0 \mathrm{a}$ \\
\hline $\mathrm{Pr}+{ }^{7} \mathrm{MgSO}_{4}$ & $100+500$ & $2,6 \mathrm{a}$ & $1,9 \mathrm{~b}$ & $1,7 \mathrm{~b}$ & $1,7 \mathrm{a}$ & $1,7 \mathrm{ab}$ & $1,7 \mathrm{a}$ \\
\hline $\mathrm{Pr}+{ }^{8}\left(\mathrm{NH}_{2}\right)_{2} \mathrm{CO}$ & $100+300$ & $2,4 \mathrm{ab}$ & $1,8 \mathrm{~b}$ & $1,6 \mathrm{~b}$ & $1,6 \mathrm{a}$ & $1,9 \mathrm{ab}$ & $1,9 \mathrm{a}$ \\
\hline $\mathrm{Pr}+\mathrm{ZnCl}_{2}+\mathrm{MnCl}_{2}+\mathrm{MgSO}_{4}$ & $100+50+50+500$ & $2,4 a b$ & $1,6 \mathrm{~b}$ & $1,6 \mathrm{~b}$ & $1,7 \mathrm{a}$ & $1,8 \mathrm{ab}$ & $1,8 \mathrm{a}$ \\
\hline $\mathrm{Pr}+\mathrm{KH}_{2} \mathrm{PO}_{3}+\left(\mathrm{NH}_{2}\right)_{2} \mathrm{CO}+\mathrm{ZnCl}_{2}$ & $100+150+300+50$ & $2,4 a b$ & $1,6 \mathrm{~b}$ & $1,6 \mathrm{~b}$ & $1,70 \mathrm{a}$ & $1,7 \mathrm{ab}$ & $1,7 \mathrm{a}$ \\
\hline Testemunha (água destilada) & - & $2,6 \mathrm{a}$ & $2,5 \mathrm{a}$ & $2,3 \mathrm{a}$ & $1,6 \mathrm{a}$ & $1,6 \mathrm{~b}$ & $1,9 \mathrm{a}$ \\
\hline Teste F & & $5,2 * *$ & $25,5^{* *}$ & $151,4 * *$ & $1,7^{\mathrm{NS}}$ & $2,2^{*}$ & $1,6^{\mathrm{NS}}$ \\
\hline CV $(\%)$ & & 6,2 & 8,9 & 3,1 & 7,4 & 11,2 & 11,4 \\
\hline
\end{tabular}

NS - não significativo; $(* *)$ Significativo a $1 \%$ de probabilidade; ${ }^{1}$ Dados originais transformados em $\ln (\mathrm{x}+5) .{ }^{2}$ Dias após o tratamento (DAT).${ }^{3}$ Letras iguais na mesma coluna não diferem entre si, pelo teste de Tukey $(p<0,05) .{ }^{4}$ Cloreto de zinco; ${ }^{5} \mathrm{Cloreto}$ de manganês; ${ }^{6}$ Fosfito de potássio; ${ }^{7}$ Sulfato de magnésio e ${ }^{8}$ Ureia. 
TABELA 4 - Número médio de ácaros vivos Brevipalpus phoenicis para as diferentes caldas do acaricida acrinathrin com adição de fertilizantes foliares.

\begin{tabular}{|c|c|c|c|c|c|c|c|}
\hline \multirow{3}{*}{ Tratamentos } & \multirow{3}{*}{$\begin{array}{c}\text { Dosagens } \\
\text { g ou } \mathrm{mL} \text { p.c./100 L }\end{array}$} & \multicolumn{6}{|c|}{ Média de ácaros ${ }^{1}$} \\
\hline & & \multicolumn{3}{|c|}{ Vivos } & \multicolumn{3}{|c|}{ Retidos na cola } \\
\hline & & $1 \mathbf{D A T}^{2}$ & 3 DAT & 5 DAT & 1 DAT & 3 DAT & 5 DAT \\
\hline Acrinathrin (Act) & 10 & $1,8 b^{3}$ & $1,6 \mathrm{~b}$ & $1,6 \mathrm{~b}$ & $2,5 \mathrm{ab}$ & $2,6 \mathrm{a}$ & $2,6 \mathrm{a}$ \\
\hline Act $+{ }^{4} \mathrm{ZnCl}_{2}$ & $10+50$ & $1,9 \mathrm{~b}$ & $1,6 \mathrm{~b}$ & $1,6 \mathrm{~b}$ & $2,2 \mathrm{c}$ & $2,4 \mathrm{a}$ & $2,4 \mathrm{a}$ \\
\hline Act $+{ }^{5} \mathrm{MnCl}_{2}$ & $10+50$ & $1,7 \mathrm{~b}$ & $1,7 \mathrm{~b}$ & $1,6 \mathrm{~b}$ & $2,6 \mathrm{a}$ & $2,6 \mathrm{a}$ & $2,6 \mathrm{a}$ \\
\hline $\mathrm{Act}+{ }^{6} \mathrm{KH}_{2} \mathrm{PO}_{3}$ & $10+150$ & $1,7 \mathrm{~b}$ & $1,7 \mathrm{~b}$ & $1,7 \mathrm{~b}$ & $2,5 \mathrm{ab}$ & $2,5 \mathrm{a}$ & $2,5 \mathrm{a}$ \\
\hline Act $+{ }^{7} \mathrm{MgSO}_{4}$ & $10+500$ & $1,9 \mathrm{~b}$ & $1,7 \mathrm{~b}$ & $1,6 \mathrm{~b}$ & $2,4 \mathrm{abc}$ & $2,5 \mathrm{a}$ & $2,5 \mathrm{a}$ \\
\hline $\mathrm{Act}+{ }^{8}\left(\mathrm{NH}_{2}\right)_{2} \mathrm{CO}$ & $10+300$ & $1,7 \mathrm{~b}$ & $1,7 \mathrm{~b}$ & $1,6 \mathrm{~b}$ & $2,3 \mathrm{bc}$ & $2,3 \mathrm{a}$ & $2,3 \mathrm{a}$ \\
\hline $\mathrm{Act}+\mathrm{ZnCl}_{2}+\mathrm{MnCl}_{2}+\mathrm{MgSO}_{4}$ & $10+50+50+500$ & $1,7 \mathrm{~b}$ & $1,6 \mathrm{~b}$ & $1,6 \mathrm{~b}$ & $2,6 \mathrm{a}$ & $2,5 \mathrm{a}$ & $2,5 \mathrm{a}$ \\
\hline $\mathrm{Act}+\mathrm{KH}_{2} \mathrm{PO}_{3}+\left(\mathrm{NH}_{2}\right)_{2} \mathrm{CO}+\mathrm{ZnCl}_{2}$ & $210+150+300+50$ & $1,8 \mathrm{~b}$ & $1,6 \mathrm{~b}$ & $1,6 \mathrm{~b}$ & $2,6 \mathrm{a}$ & $2,6 \mathrm{a}$ & $2,6 \mathrm{a}$ \\
\hline Testemunha (água destilada) & - & $2,6 \mathrm{a}$ & $2,4 \mathrm{a}$ & $2,4 \mathrm{a}$ & $1,8 \mathrm{~d}$ & $1,8 \mathrm{~b}$ & $1,8 \mathrm{~b}$ \\
\hline Teste F & & $20,9 * *$ & $41,4 * *$ & $242,8 * *$ & $26,0 * *$ & $14,1 * *$ & $14,1 * *$ \\
\hline CV $(\%)$ & & 9,4 & 6,6 & 2,9 & 6,1 & 7,2 & 7,2 \\
\hline
\end{tabular}

NS - não significativo; $(* *)$ Significativo a $1 \%$ de probabilidade; ${ }^{1}$ Dados originais transformados em $\ln (\mathrm{x}+5) .{ }^{2}$ Dias após o tratamento (DAT).${ }^{3}$ Letras iguais na mesma coluna não diferem entre si, pelo teste de Tukey $(\mathrm{p}<0,05) .{ }^{4}$ Cloreto de zinco; ${ }^{5} \mathrm{Cloreto}$ de manganês; ${ }^{6}$ Fosfito de potássio; ${ }^{7}$ Sulfato de magnésio e ${ }^{8}$ Ureia.

TABELA 5 - Eficiência de controle (\%) de ácaros Brevipalpus phoenicis nos diferentes bioensaios de avaliação de caldas acaricidas com adição de fertilizantes foliares.

\section{Tratamentos}

Eficiência de controle (\%)

Cyhexatin Propargite Acrinathrin

1 DAT $^{2} 3$ DAT 1 DAT 3 DAT 5 DAT 1 DAT 3 DAT 5 DAT

Acaricida $^{1}$

Acaricida $+{ }^{3} \mathrm{ZnCl}_{2}$

Acaricida $+{ }^{4} \mathrm{MnCl}_{2}$

Acaricida $+{ }^{5} \mathrm{KH}_{2} \mathrm{PO}_{3}$

Acaricida $+{ }^{6} \mathrm{MgSO}_{4}$

Acaricida $+{ }^{7}\left(\mathrm{NH}_{2}\right)_{2} \mathrm{CO}$

Acaricida $+\mathrm{ZnCl}_{2}+\mathrm{MnCl}_{2}+\mathrm{MgSO}_{4}$

Acaricida $+\mathrm{KH}_{2} \mathrm{PO}_{3}+\left(\mathrm{NH}_{2}\right)_{2} \mathrm{CO}+\mathrm{ZnCl}_{2} \quad 100,0 \quad 100,0 \quad 26,8 \quad 98,3 \quad 100,0 \quad 43,1 \quad 100,0 \quad 100,0$

Testemunha (água destilada)

$96,9 \quad 100,0 \quad 5,2 \quad 94,4 \quad 100,0 \quad 64,6 \quad 92,9 \quad 100,0$

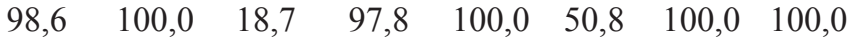

$94,5 \quad 100,0 \quad 42,0 \quad 98,4 \quad 100,0 \quad 55,6 \quad 75,0 \quad 100,0$

$\begin{array}{llllllll}95,0 & 100,0 & 23,7 & 82,4 & 100,0 & 55,2 & 73,9 & 88,1\end{array}$

$\begin{array}{llllllll}87,6 & 100,0 & 7,8 & 77,0 & 94,1 & 12,5 & 60,9 & 97,2\end{array}$

$\begin{array}{llllllll}95,4 & 100,0 & 35,8 & 90,7 & 100,0 & 91,9 & 89,8 & 100,0\end{array}$

$100,0 \quad 100,0 \quad 22,0 \quad 98,1 \quad 100,0 \quad 50,0 \quad 87,5 \quad 87,5$

${ }^{1}$ Cyhexatin, propargite ou acrinathrin; ${ }^{2}$ Dias após o tratamento (DAT); ${ }^{3}$ Cloreto de zinco; ${ }^{4}$ Cloreto de manganês; ${ }^{5}$ Fosfito de potássio; ${ }^{6}$ Sulfato de magnésio e ${ }^{7}$ Ureia. 
TABELA 6 - Valores de pH, condutividade elétrica (Cond.), temperatura (Temp.) e oxigênio dissolvido $\left(\mathrm{O}_{2}\right)$ de diferentes caldas acaricidas.

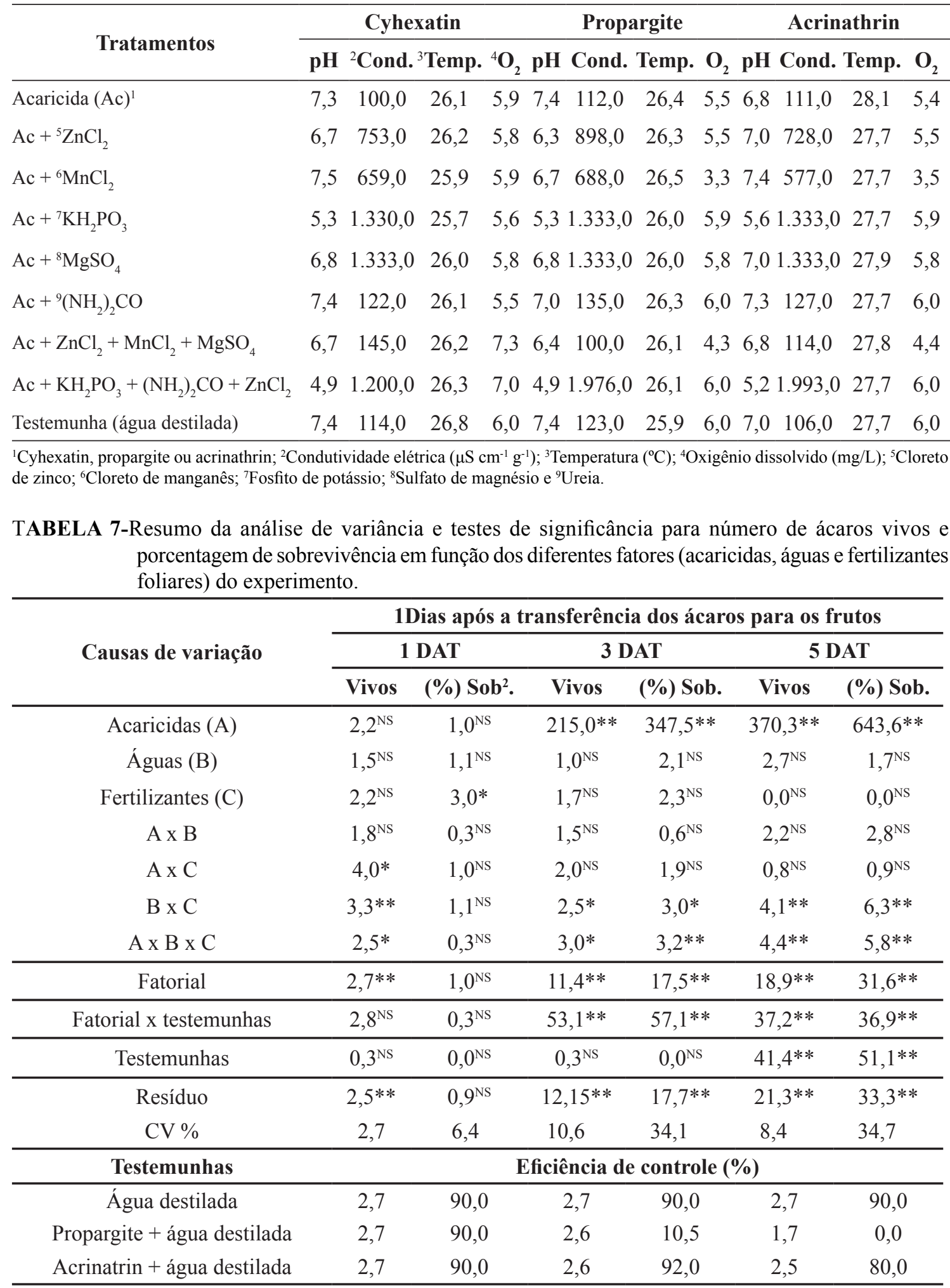

NS - não significativo ; $(* *)$ significativo a $1 \%$ e a $(*) 5 \%$ de probabilidade; transformados $\ln (\mathrm{x}+5)$;

${ }^{1}$ Teste F; ${ }^{2}$ Porcentagem de sobrevivência transformada em Arc Seno [raiz (\% Sobrev./100)]. 
TABELA 8 - Médias das porcentagens de sobrevivências de ácaros Brevipalpus phoenicis nas avaliações realizadas a 1; 3 e 5 dias após a aplicação, nas interações dos fatores água de diferentes locais e fertilizantes foliares.

\begin{tabular}{|c|c|c|c|c|}
\hline \multirow[b]{2}{*}{ Águas } & \multicolumn{4}{|c|}{1 dia após a aplicação } \\
\hline & ${ }^{1}$ S/ Fert. & ${ }^{2} \mathrm{KH}_{2} \mathrm{PO}_{3}$ & ${ }^{3} \mathrm{MgSO}_{4}$ & $\begin{array}{c}{ }^{4} \mathrm{ZnCl}_{2}+{ }^{5} \mathrm{MnCl}_{2} \\
+\mathrm{MgSO}_{4}\end{array}$ \\
\hline Itápolis & 90,0 aA & $81,5 \mathrm{bB}$ & $90,0 \mathrm{aA}$ & $90,0 \mathrm{aA}^{4}$ \\
\hline Pirangi & $90,0 \mathrm{aA}$ & $90,0 \mathrm{aA}$ & $90,0 \mathrm{aA}$ & $90,0 \mathrm{aA}$ \\
\hline Pirassununga & $90,0 \mathrm{aA}$ & $86,5 \mathrm{aB}$ & $90,0 \mathrm{aA}$ & $90,0 \mathrm{aA}$ \\
\hline \multirow[b]{2}{*}{ Águas } & \multicolumn{4}{|c|}{3 dias após a aplicação } \\
\hline & S/ Fert. & $\mathrm{KH}_{2} \mathrm{PO}_{3}$ & $\mathrm{MgSO}_{4}$ & $\begin{array}{c}\mathrm{ZnCl}_{2}+\mathrm{MnCl}_{2} \\
+\mathrm{MgSO}_{4}\end{array}$ \\
\hline Itápolis & 47,3 aAB & $65,5 \mathrm{aA}$ & $44,5 \mathrm{aAB}$ & $28,6 \mathrm{aB}$ \\
\hline Pirangi & 61,2 aA & $36,0 \mathrm{bB}$ & $45,0 \mathrm{aAB}$ & $45,0 \mathrm{aAB}$ \\
\hline Pirassununga & $45,0 \mathrm{aA}$ & $65,5 \mathrm{aA}$ & 55,3 aA & 47,6 aA \\
\hline \multirow[b]{2}{*}{ Águas } & \multicolumn{4}{|c|}{5 dias após a aplicação } \\
\hline & S/ Fert. & $\mathrm{KH}_{2} \mathrm{PO}_{3}$ & $\mathrm{MgSO}_{4}$ & $\begin{array}{c}\mathrm{ZnCl}_{2}+\mathrm{MnCl}_{2} \\
+\mathrm{MgSO}_{4}\end{array}$ \\
\hline Itápolis & 42,2 aA & $28,8 \mathrm{aA}$ & $37,3 \mathrm{abA}$ & $18,3 \mathrm{bB}$ \\
\hline Pirangi & $41,7 \mathrm{aA}$ & $28,8 \mathrm{aA}$ & $27,6 \mathrm{bA}$ & $45,0 \mathrm{aA}$ \\
\hline Pirassununga & $24,7 \mathrm{bB}$ & $45,0 \mathrm{aA}$ & $45,0 \mathrm{aA}$ & $45,0 \mathrm{aA}$ \\
\hline
\end{tabular}

Letra minúscula na coluna e maiúscula na linha, não diferem entre si, pelo teste de Tukey,

a $5 \%$ de probabilidade. ${ }^{1}$ Sem adição de fertilizante foliar. ${ }^{2}$ Fosfito de potássio; ${ }^{3}$ Sulfato de magnésio; ${ }^{4}$ Cloreto de zinco e ${ }^{5}$ Cloreto de manganês.

TABELA 9- Eficiência de controle (\%) de ácaros Brevipalpus phoenicis nos diferentes tratamentos nas avaliações realizadas a 1; 3 e 5 dias após a aplicação dos produtos.

\begin{tabular}{|c|c|c|c|c|c|c|}
\hline \multirow[b]{2}{*}{ Tratamentos } & \multicolumn{3}{|c|}{ Fatores } & \multicolumn{3}{|c|}{ Eficiência de controle (\%) } \\
\hline & Acaricida & Águas & Fertilizante & 1 DAT & 3 DAT & 5 DAT \\
\hline 1 & Propargite & Itápolis & $\mathrm{S} /$ Fertilizante & 0,0 & 97,5 & 100,0 \\
\hline 2 & Propargite & Itápolis & $\mathrm{KH}_{2} \mathrm{PO}$ & 13,9 & 63,9 & 100,0 \\
\hline 3 & Propargite & Itápolis & $\mathrm{MgSO}_{4}$ & 0,0 & 90,6 & 100,0 \\
\hline 4 & Propargite & Itápolis & $\mathrm{ZnCl}_{2}+\mathrm{MnCl}_{2}+\mathrm{MgSO}_{4}$ & 0,0 & 97,5 & 100,0 \\
\hline 5 & Propargite & Pirangi & S/ Fertilizante & 0,0 & 58,2 & 88,8 \\
\hline 6 & Propargite & Pirangi & $\mathrm{KH}_{2} \mathrm{PO}$ & 0,0 & 97,5 & 100,0 \\
\hline 7 & Propargite & Pirangi & $\mathrm{MgSO}_{4}^{3}$ & 0,0 & 100,0 & 100,0 \\
\hline 8 & Propargite & Pirangi & $\mathrm{ZnCl}_{2}+\mathrm{MnCl}_{2}+\mathrm{MgSO}_{4}$ & 0,0 & 100,0 & 100,0 \\
\hline 9 & Propargite & Pirassununga & S/ Fertilizante & 0,0 & 100,0 & 100,0 \\
\hline 10 & Propargite & Pirassununga & $\mathrm{KH}_{2} \mathrm{PO}_{3}$ & 5,6 & 55,6 & 100,0 \\
\hline 11 & Propargite & Pirassununga & $\mathrm{MgSO}_{4}$ & 0,0 & 83,3 & 100,0 \\
\hline 12 & Propargite & Pirassununga & $\mathrm{ZnCl}_{2}+\mathrm{MnCl}_{2}+\mathrm{MgSO}_{4}$ & 0,0 & 96,9 & 100,0 \\
\hline 13 & Acrinathrin & Itápolis & S/ Fertilizante & 0,0 & 0,0 & 3,6 \\
\hline 14 & Acrinathrin & Itápolis & $\mathrm{KH}_{2} \mathrm{PO}_{3}$ & 2,8 & 6,3 & 12,5 \\
\hline 15 & Acrinathrin & Itápolis & $\mathrm{MgSO}_{4}$ & 0,0 & 11,1 & 19,4 \\
\hline 16 & Acrinathrin & Itápolis & $\mathrm{ZnCl}_{2}+\mathrm{MnCl}_{2}+\mathrm{MgSO}_{4}$ & 0,0 & 37,8 & 63,8 \\
\hline 17 & Acrinathrin & Pirangi & S/ Fertilizante & 0,0 & 12,5 & 22,2 \\
\hline 18 & Acrinathrin & Pirangi & $\mathrm{KH}_{2} \mathrm{PO}_{3}$ & 0,0 & 25,0 & 35,0 \\
\hline 19 & Acrinathrin & Pirangi & $\mathrm{MgSO}_{4}$ & 0,0 & 0,0 & 39,3 \\
\hline 20 & Acrinathrin & Pirangi & $\mathrm{ZnCl}_{2}+\mathrm{MnCl}_{2}+\mathrm{MgSO}_{4}$ & 0,0 & 0,0 & 0,0 \\
\hline 21 & Acrinathrin & Pirassununga & S/ Fertilizante & 0,0 & 0,0 & 43,3 \\
\hline 22 & Acrinathrin & Pirassununga & $\mathrm{KH}_{2} \mathrm{PO}_{3}$ & 0,0 & 0,0 & 0,0 \\
\hline 23 & Acrinathrin & Pirassununga & $\mathrm{MgSO}_{4}^{3}$ & 0,0 & 0,0 & 0,0 \\
\hline 24 & Acrinathrin & Pirassununga & $\mathrm{ZnCl}_{2}+\mathrm{MnCl}_{2}+\mathrm{MgSO}_{4}$ & 0,0 & 0,0 & 0,0 \\
\hline 25 & Propargite & Água destilada & - & 0,0 & 90,0 & 96,4 \\
\hline 26 & Acrinathrin & Água destilada & - & 0,0 & 7,8 & 20,1 \\
\hline 27 & - & Água destilada & - & - & - & - \\
\hline
\end{tabular}




\section{CONCLUSÕES}

1-Os fertilizantes foliares cloreto de zinco, cloreto de manganês, ureia e a mistura fosfito de potássio + ureia + cloreto de zinco não afetam a ação dos acaricidas cyhexatin, propargite e acrinathrin sobre o controle de Brevipalpus phoenicis, sendo sua utilização pelo citricultor, condicional à legislação em vigor e às possíveis implicações fitotóxicas aos citros ou outras inter-relações com organismos- não alvo.

2-O fosfito de potássio, o sulfato de magnésio e a mistura dos cloretos de zinco e de manganês com o sulfato de magnésio resultam em diminuição na porcentagem de eficiência dos acaricidas propargite e acrinathrin sobre B. phoenicis.

3-As águas provenientes dos municípios de Itápolis, Pirangi e Pirassununga, juntamente com fertilizantes foliares, interferem na ação dos acaricidas propargite e acrinathin no controle de $B$. phoenicis, sendo a água de Itápolis a que apresenta resultados melhores em termos de eficiência.

\section{REFERÊNCIAS}

ABBOTT, W.S. A method of computing the effectiveness of an insecticide. Journal of Economic Entomology, College Park, v.18, p.265-7, 1925.

AGRIANUAL: anuário estatístico da agricultura brasileira. São Paulo: FNP Consultoria e Comércio, 2010. 520p.

ALBUQUERQUE, F.A.; OLIVEIRA, C.A.L.; BARRETO, M. Estudos da relação entre as incidências de verrugose da laranja-doce e leprose dos citros em frutos de laranja pêra, Científica, Jaboticabal, v.25, p.393-402, 1997.

AL-MUGHRABI, K.I.; NAZER, I.K. Chemistry of King Canal water in the Jordan Valley. II. Effect of water $\mathrm{pH}$ on the stability of Dimetoato. Arabian Gulf Science, Dhahran, v.9, n.2, p.13-22, 1991.

ANDRADE, T.L.C. Ação acaricida do hexythiazox, dicofol e óxido de fenbutatin, em três níveis de pH da calda. 1997. 71f. Dissertação (Mestrado em Agronomia) - Faculdade de Ciências Agrárias e Veterinárias de Jaboticabal, Universidade Estadual Paulista, Jaboticabal, 1997.
BASTIANEL, M.; NOVELLI, V.M.; KITAJIMA, E.W.; KUBO, K.S.; BASSANEZI, R.B.; MACHADO, M.A.; FREITAS-ASTÚA, J.F. Citrus leprosis: centennial of an unusual mite virus pathosystem. Plant Disease, Saint Paul, v.94, n.3, p.284-292, 2010.

BAUR, J.R.; BOVEY, R.W.; BAKER, R.D.; RILEY, I. Absorption and penetration of picloram and 2,4,5$\mathrm{T}$ into detached live oak leaves. Weed Science, Champaign, v.19, p.138-141, 1971.

BETANA, M.D.; HUSSEIN, M.A.; EL-KADI, A.M.A. Influence of some adjuvants on physicochemical properties, effictiveness, and persistence of some insecticides formulations. Journal of Agricultural Science, Mansoura, v.29, n.4, p.21052115, 2004.

BOtEON, M.; PAGLIUCA, L.G. Análise da sustentabilidade econômica da citricultura paulista. Citrus Research \& Technology, Cordeirópolis, v.31, n.2, p.101-106, 2010.

BRASIL. Decreto No 4074, de 04 de janeiro de 2002. Diário Oficial da União, Brasília, DF, 04 jan. 2002. Seção I.

BRASIL. Instrução Normativa 05 de 23 de fevereiro de 2007. Diário Oficial da União, Brasília, DF, 01 mar. 2007. Seção 1.

CAMPOS NETO H.H; MOURA, E.; PASSOS, H.R.; CINIGLIO NETO F.; MARICONI, F.A.M.; SCARPARI FILHO, J.A. Combate experimental ao ácaro da leprose Brevipalpus phoenicis (Geijskes, 1939) em citros. Scientia Agricola, Piracicaba, v.50, n.2, p. 267-271, 1993.

EL-ATTAL, Z.M.; MOUSTAFA, O.K.; DIAB, S.A. Influence of foliar fertilizers on the toxicity and tolerance to some insecticides in the cotton leafworm. The Journal of Agricultural Science, Cambridge, n.102, p.111-114, 1984.

EL-SISI,A.G.; RADWAN, S.M.E.; A.EL-HAMAKY, M. Effect of spray irrigation on the residual activity of insecticides. Agricultural Research Review, Cairo, v.67, n.1, p.13-18, 1989.

FARGHALY, S.F.; TORKEY, H.M; ABOUYOUSEF, H.M. Natural Extracts and Their Chemical Constituents in Relation to Toxicity Against Whitefly (Bemisia tabaci) and Aphid (Aphis craccivora). Australian Journal of Basic and Applied Sciences, Austrália, v.3, n.4, p.3217-3223, 2009. 
HOUGHTON, R.D. Pesticide compatibility: an overview from technical services. ASTM Special Technical Publication, Philadelphia, n.764, p.310,1982

HUSSEIN, M.A. Improvement of insecticidal performance using certain additives against cotton leafworm, Spodoptera littoralis. Journal of Agricultural Science, Mansoura, v.27, n.9, p.64176426, 2002.

JONES, J.P.; KELSHEIMER, E.G. The compatibility of several pesticides and nutrients on tomato. Florida: Florida Agricultural Experiment Station, 1968. p.167-172.

KITAJIMA, E.W.; RODRIGUES, J.C.V.; FREITASASTÚA, J. An annotated list of ornamentals naturally found infected by Brevipalpus mite-transmitted viruses. Scientia Agricola, Piracicaba, v.67, n.3, p.348-371, 2010.

MATUO, T.K.; MATUO, T. Efeito da pressurização com $\mathrm{CO} 2$ sobre o $\mathrm{pH}$ da água. Planta Daninha, Botucatu, v.13, n.1, 1995.

MOXNESS, K.D.; LYM, R.G. Environment and spray additive effects on picloram absorption and translocation in leafy spurge (Euphorbia esula). Weed Science, Champaign, v. 37, p. 181-186, 1989.

PRADO, E.P.; ARAÚJO, D.; RAETANO, C.G.; DAL POGETTO, M.H.F.A.; AGUIAR-JÚNIOR, H.O.; CHRISTOVAM, R.S. Influência da dureza e potencial hidrogeniônico da calda de pulverização sobre o controle do ácaro-da-leprose em frutos de laranja doce. Bragantia, Campinas, v.70, n.2, p.389396, 2011.

RADWAN, S.M.E.; EL-SISI, A.G.; MAKADEY, M.A.; HAMOUDA, S.H. Using local additives for improving the efficiency of pesticides against Spodoptera littoralis (Boisd.). Minia Journal of Agricultural Research and Development, Cairo, v.16, n.4, p.997-999, 1994.
REEVES, B. The effect of water $\mathrm{pH}$ on pesticides. Oregon Horticultural Society, Oregon, v.74, p.138142,1983

SAYED, M.R.; FAYEY, A.B.; MANSOUR, A.A. Biochemical and histopathological effect of crude extracts on Spodoptera littoralis larvae. Journal of Evolutionary Biology Research, Oxford, v.3, n.5, p.67-78, 2011.

SCHENCK, N.C.; ADLERZ, W.C. Compatibility of insecticides, fungicides, and foliar fertilizers on watermelon. Flórida: Florida Agricultural Experiment Station, 1962. p.209-212. (Circular)

SIMKISS, K.; MASON, A.Z. Metal ions: metabolic and toxic effects. In: HOCHACHKA, P.W. (Ed.). The mollusca. New York: Academic Press, 1983. v.2, p.162-172.

TAWFIK, M.H.; EL-SISI, A.G. The effect of mixing some foliar fertilizers on the physical properties and insecticidal activity of some locally spray oils against the scale insect Parlatoria ziziphus. In: NATIONAL CONFERENCE OF PESTS AND DISEASE OF VEGETABLES AND FRUITS, 2., 1987, Ismallia. Proceedings... p.367-376 .

TUNDISI, J.G. Typology if reservoirs in southern Brazil. E. Schweizerbart'sche Verlagsbuchhandlung, Stuttgart, v.21, n.1, p.10311039, 1981.

WRIGHT, J.F.; LINDSAY, A.D.; SAWYER, E. Pesticide tank mix applications: first Conference. Philadelfia: ASTM, 1982. 